\title{
Diagnostic and Therapeutic Resources for Risk Stratification of Patients With Acute Coronary Syndrome
}

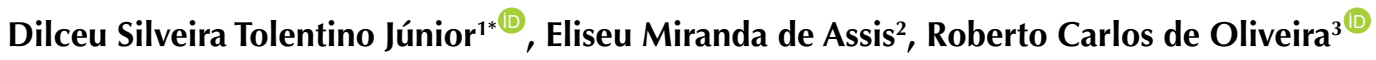 \\ 'Faculty of Medicine, Federal University of the Jequitinhonha and Mucuri Valleys, Teófilo Otoni, Minas Gerais, Brazil \\ ${ }^{2}$ Academic Department, Federal Institute of Bahia, Eunápolis, Bahia, Brazil \\ ${ }^{3}$ Vale do Rio Doce University, Health, Individual and Society Research Center, Governador Valadares, Minas Gerais, \\ Brazil
}

*Corresponding Author: Dilceu Silveira Tolentino Júnior, M.D., Professor, Faculty of Medicine, Federal University of the Jequitinhonha and Mucuri Valleys, Teófilo Otoni, Minas Gerais, Brazil. Tel: +55-33988431523, Email: dilceujunior@ bol.com.br

Received September 20, 2020; Accepted January 14, 2021; Online Published February 13, 2021

\begin{abstract}
Acute coronary syndrome (ACS) results from acute obstruction of a coronary artery which is responsible for a high mortality rate worldwide. The consequences depend on the degree and location of the obstruction and vary from unstable angina to non-ST segment elevation myocardial infarction (NSTEMI), ST-segment elevation myocardial infarction, and sudden cardiac death. The symptoms are similar in each of these syndromes (except for sudden death), involving chest discomfort with or without dyspnea, nausea, and diaphoresis. The diagnosis is possible; thanks to the electrocardiogram that is essential and the existence or absence of serological markers. In addition to these initial resources, other diagnostic methods are noteworthy, such as stress electrocardiogram, echocardiography, nuclear cardiology, computed tomography angiography, and exercise test. Other necessary measures are the stratification of the identified cases according to the degree of risk, availability of a coronary intensive care unit, and the establishment of the opportune treatment that consists of oxygen therapy, analgesia, sedation, antiplatelet, anticoagulants, nitrates, beta-blockers drugs, reperfusion of emergency with fibrinolytic drugs, percutaneous intervention or, occasionally, myocardial revascularization surgery to provide the recovery and consequently a better quality of life for the patient. This brief review aims to discuss the available diagnostic and therapeutic resources and the appropriate risk stratification for adequate care for the victims of acute coronary heart disease promptly in a hospital setting.

Keywords: Diagnosis, Treatment, Cardiovascular Risk Stratification, Coronary Disease
\end{abstract}

\section{Background}

In Brazil, it is estimated that 300000 to 400000 cases of infarction occur annually, and that of every 5 to 7 cases, one death occurs. Thus, despite the numerous therapeutic and diagnostic resources obtained in recent decades, ACS is still one of the most important causes of morbidity and mortality in our country. ${ }^{1}$

Most deaths from AMI worldwide occur in the first hours of the disease onset, with $40 \%$ to $65 \%$ of cases in the first hour and approximately $80 \%$ in the first 24 hours. Thus, patients with ACS must be treated promptly. ${ }^{2}$ A large part of deaths could have been prevented if they had been taken in due time to a hospital unit that adequately would manage this dreadful event through a specialized team that appropriates diagnostic, therapeutic resources, and risk classification so that the propaedeutics is adequate to stabilize these conditions which are often challenging. ${ }^{3}$

The obstruction and consequent reduction in coronary flow are commonly due to the atheroma physical disruption with the posterior constitution of obliterating clots. Coronary vasoconstriction accompanied by microembolization may also be involved in this process. The clinical presentation of coronary artery disease (CAD) can be identified in its chronic form, as stable angina, and in the acute form, as acute coronary syndromes (ACSs), with or without ST-segment elevation. ${ }^{2}$

A detailed interview associated with clinical consultation performs a key function in the risk classification of individuals who have unstable myocardial ischemic syndromes without ST-segment elevation. ${ }^{1}$ The risk stratification approach has been widely utilized, and advances have occurred in accordance with the standards suggested by several scholars on the subject, mainly with regard to the quantification of troponin, allowing for efficient classification, especially of those patients with the insufficient arterial flow. ${ }^{2}$ This brief review aims to

Copyright (C) 2021 The Author(s). This is an open-access article distributed under the terms of the Creative Commons Attribution License (http:// creativecommons.org/licenses/by/4.0), which permits unrestricted use, distribution, and reproduction in any medium, provided the original work is properly cited. 
discuss the resources currently available for adequate care for victims of acute coronary heart disease in a timely manner, with crucial treatment as well as invasive and noninvasive diagnostic methods that make all the differences in reducing mortality and risks arising from these morbid conditions.

\section{The Use of Electrocardiographic Resources}

Regardless of the lesser precision to distinguish acute myocardial infarction (AMI) in its inner third extending across the entire thickness of the endocardial wall in AMI (through the Q wave), the electrocardiogram is essential for the diagnosis of patients with unstable myocardial ischemic syndromes non-ST segment elevation. ${ }^{2}$ Momentary changes that occur in the aforementioned syndrome, such as inversion of the $\mathrm{T}$ wave, constitute relevant predictive indicators for the occurrence of early mortality or obstruction of arterial circulation. Generally, when the result of the electrocardiographic examination indicates no changes, this fact should not eliminate the investigation of unstable myocardial ischemic syndromes without ST-segment elevation. ${ }^{3}$

\section{Diagnostic Resources of Biological and Chemical Markers of Myocardial Necrosis}

New biological and chemical markers are essential instruments in the investigation and prediction of individual cases who present unstable myocardial ischemic syndromes without ST-segment elevation. ${ }^{4}$

These markers must be interpreted in a way associated with the individual's health condition and the result of the electrocardiogram since several non-coronary situations can lead to elevation. ${ }^{5}$

Levels of necrosis markers after percutaneous transluminal coronary angioplasty or myocardial revascularization surgery indicate AMI when the increase is 5 to 10 times greater than the reference value (postPCI and post-CRM, respectively) in conjunction with symptoms, electrocardiogram changes, and imaging tests. ${ }^{5}$ Some muscle fiber proteins and cardiac enzymes are released in the myocardial injury due to their high negative scores suggestive after the sixth hour of symptoms, which can be considered in early discharge protocols in the emergency room. ${ }^{6}$

The stress electrocardiogram, also known as an exercise stress test, aims to recognize possible partial or total blockage of the cardiac arteries, to predict and subsidize appropriate therapeutic measures in response to unstable myocardial ischemic syndromes without ST-segment elevation. However, the test should not be used to screen for ischemic disease in asymptomatic individuals, since in this context, the test has low diagnostic accuracy; generating false-positive results, dispensable additional propaedeutics, incorrect treatments with unnecessary costs, and risks. ${ }^{7}$

\section{Different Types of Diagnostic Resources Used}

Echocardiography is very useful to individuals who have NSTEMI. ${ }^{8}$ The indication of the echocardiographic study to evaluate the left ventricular systolic function corresponds to one of the main clinical applications of this diagnostic method. Ventricular contractility represents the result of a complex interaction between the contractile state of the cardiac muscle and the pre-and afterload levels. ${ }^{8}$

Nuclear cardiology is important for individuals who have STEMI, since the information obtained through scintigraphy allows the assessment of myocardial perfusion according to the location of coronary irrigation. Myocardial scintigraphy with physical or pharmacological stress is performed in patients with unstable myocardial ischemic syndromes of low or intermediate risk after resolution of a heightened condition. ${ }^{9}$ In the context of a critical care room or acute pain, the injection of the radiopharmaceutical should be performed only at rest, while the patient is still symptomatic, and the images must be obtained within six hours. If the patient is considered to be of low risk, a very low probability of subsequent cardiac events is determined. ${ }^{10}$

Computed tomography angiography of the coronary arteries constitutes a relevant resource in the evaluation of individuals with clinical manifestation suggestive of recent thoracic pain, especially in low and medium risk individuals, being an assured procedure that allows investigation of unstable myocardial ischemic, which reduces hospitalization time and, eventually, the total cost. $^{11}$

\section{Therapeutic Resources Used for the Treatment of Coronary Syndromes}

Studies have shown that the use of oxygen-based treatment is capable of containing the progression of the obstructive lesion. ${ }^{12}$ Its use is generally maintained until the maximum period of four hours after the end of the pain discomfort. The use of oxygen inadvertently causes an increase in the formation of reactive oxygen species, paradoxical tissue hypoxia, and absorption atelectasis. ${ }^{3}$

The treatment of pain decreases the consumption of oxygen by the ischemic myocardium, caused by the activation of the sympathetic nervous system. Analgesia should preferably be done with intravenous morphine sulfate - except for patients allergic to this drug - at the initial dose of 2.0-8.0 mg (usually sufficient to relieve pain and anxiety). With blood pressure monitoring, these doses can be repeated at intervals of 5-15 minutes. ${ }^{13}$ In case of non-availability or hypersensitivity to the drug, morphine sulfate can be replaced by meperidine sulfate, in fractional doses of 20-50 mg. ${ }^{3}$

Nitrates can cause vasodilation of the epicardial arteries even when affected by atherosclerosis. It mainly produces a decrease in myocardial oxygen demand indirectly through its hemodynamic effect. ${ }^{14,15}$ The reduction in volume and the final diastolic pressure of the ventricles lead to a reduction in the tension of the ventricular wall. . The 
tension of the wall is one of the main determinants of the level of oxygen consumption by the myocardial fiber and its reduction seems to be the main antianginal mechanism of nitrates.

In the absence of contraindications, adrenergic betablockers should be started immediately, preferably orally, after the patient's admission. To date, there are no data on the systematic evaluation of its use in the prehospital phase. A study has demonstrated lower rates of $\mathrm{Q}$ wave AMI, congestive heart failure, and ventricular fibrillation, without a marked increase in side effects. ${ }^{16}$

The effectiveness of the use of beta-blockers in the acute phase of myocardial infarction has been reassessed by the results of studies that have not confirmed the benefit previously described. In addition to causing more prolonged hypotension and a greater number of cases of bradycardia and cardiogenic shock, there was no reduction in mortality. Today, it should be used more carefully in venous administration. ${ }^{16}$

Calcium channel antagonists have different actions on the cardiovascular system that can be more specific and potent in the systemic arterial coronary, coronary and myocardial, or cardiac conduction system. This class of medication promotes the regression of ventricular hypertrophy and improves the diastolic performance of the left ventricle, however, some may deteriorate systolic function in the presence of myocardial insufficiency. It has been used to try to control refractory ischemic symptoms in patients already using nitrates and beta-blockers in adequate doses, or in those who do not tolerate the use of these drugs, or even in those with the syndrome of Prinzmetal. In patients with impaired left ventricular function and/or changes in atrioventricular conduction, these drugs should be avoided. ${ }^{3,17}$

Antiplatelet agents are essential once coronary thrombosis has a prominent role in triggering and progressing the symptoms of unstable myocardial ischemic syndrome (UMIS), being necessary the use of antithrombotics in the treatment of individuals who have such clinical conditions. Salicylates are excellent weak acids that cross cell membranes with relative ease and can be prescribed regularly unless the patient has an allergy to the drug or some degree of active bleeding from the gastrointestinal tract. ${ }^{18}$

Clopidogrel is indicated for adult patients as secondary prevention of recent atherothrombotic events (AMI, stroke, and vascular death or peripheral arterial disease). ${ }^{19}$ The double dose of clopidogrel was also not evaluated in patients who received thrombolytic therapy or were treated without reperfusion and should not be used in these patients. ${ }^{20}$

Antithrombinics and anticoagulants are drugs that inhibit the generation of thrombin and/or its activity. The use of anticoagulants in ACSs is an area of active investigation. ${ }^{21}$ It is difficult to reach definitive conclusions about the best anticoagulant strategy, due to different treatment times, uncertainty about equipotent anticoagulant doses, and different anti-aggregating drugs used in the studies. ${ }^{22}$ Low molecular weight heparins are obtained by depolymerizing unfractionated heparin (UFH) s and selecting those with lower molecular weights (between 2000 and $10000 \mathrm{Da}$ ). They have better subcutaneous absorption, less protein binding, less platelet activation, and a more predictable and reproducible effect. ${ }^{23,24}$

Fondaparinux is a synthetic pentasaccharide analog to the antithrombin binding site present in the heparin molecules. It acts by neutralizing the Xa factor and, thus, prevents thrombin generation. This medication has excellent bioavailability after subcutaneous injection and a plasma half-life of 17 hours, which allows its administration once a day. In short, fondaparinux proved to be safer for patients with ACSs. ${ }^{25-27}$

\section{Other Diagnostic Resources for Cardiovascular Monitoring}

Hemodynamic and cineangiocardiographic study allows direct visualization of coronary lumen, with an assessment of the extent and severity of obstructions, and analysis of systolic and diastolic ventricular functions. The examination may also include an evaluation of the functional significance of the anatomically detected lesions, by direct measurement of the fractional coronary flow reserve. ${ }^{3}$

The stress echocardiogram exam consists of intravenous administration of dobutamine, dipyridamole, with simultaneous recording of left ventricular images by echocardiogram. The main indication is in the investigation of myocardial ischemia, particularly in individuals with difficulties to perform physical exercises or individuals whose layout makes it difficult to analyze the ST segment (previous changes, conduction disorders). Another indication is in the research of myocardial viability. ${ }^{3}$

The development of contrasts containing microbubbles of smaller diameter and greater stability, associated with technological advances, such as the intermittent harmonic image and the image with low mechanical index, allowed the study of myocardial perfusion by echocardiography ${ }^{3}$

Myocardial perfusion scintigraphy is a non-invasive method developed over 30 years ago, which can demonstrate the extent and intensity of myocardial perfusion abnormalities early and with high precision, which can often have an important association with adverse events. A normal scintigraphy results in a risk of cardiac events of less than $1 \%$ per year, giving an even greater prognostic value to the method. ${ }^{3}$

Nuclear angiocardiography is obtained by synchronizing the tomographic scintigraphic study with the electrocardiogram (ECG). Thus, it is possible to assess regional systolic function and estimate ventricular ejection force, adding more diagnostic and prognostic information. ${ }^{3}$

Cardiovascular magnetic resonance is a technique based on the use of radiofrequency in tissues submitted to a magnetic field, it does not use ionizing radiation or nephrotoxic contrasts, being one of the safest methods in 
cardiovascular diagnosis. ${ }^{30}$

\section{Risk Stratification for a Better Approach to Acute Coronary Syndromes}

Risk stratification must be conducted by establishing a continuous process that includes initial clinical evaluation, availability of subsidiary exams, and complementary methods described in this review article. ${ }^{27}$ It is worth noting that the benefit observed with the "interventionist" strategy tends to be greater in the long-term follow-up when compared to the initial phase, in which, paradoxically, the risk with the use of this strategy may be greater. ${ }^{28}$

The prognosis of the ACS is very variable and the risk stratification is intended to estimate the risk of AMI and death from cardiovascular causes. Furthermore, it implies better targeting therapies for patients at higher risk for adverse cardiovascular events and also defines the best treatment strategy to avoid unnecessary interventions and adverse effects that harm low-risk patients. ${ }^{31}$

Prognostic information is obtained through careful clinical evaluation and the evolution of patients during the first days and the patient's response to anti-ischemic and antithrombotic treatment when they are admitted to the hospital. There are several risk stratification scores that can predict death or AMI in ACS. Among the most used nowadays, the GRACE and TIMI scores stand out for being feasible and accessible. ${ }^{32}$

Patients with an ACS without low-risk ST-segment elevation do not benefit from PCI, unless myocardial ischemia is found in non-invasive functional tests. ${ }^{33}$ Exercise testing (ET) is the method of choice in the evaluation of patients with low-risk AI in most cases. Imaging methods (stress echocardiography and myocardial scintigraphy) have a diagnostic performance similar or superior to TE, but with a higher cost. ${ }^{33}$

In this group, the results are similar, both in the initial conservative approach and in the invasive therapeutic strategy. The advantage of the initial conservative strategy is that several patients stabilize themselves with clinical treatment, avoiding costs and possibly unnecessary invasive procedures. ${ }^{34}$

A patient with an ACS without low-risk ST-segment elevation with an intermediate risk of a new event may be subjected to non-invasive risk stratification or invasive risk stratification. The non-invasive stress test is recommended for this patient as long as it is free of ischemia at rest or with minimal effort for at least 12 hours. ${ }^{35}$

The invasive strategy in high-risk patients reduces the rates of AMI, severe angina, and long-term rehospitalization. Such patients should go directly to angiography without a non-invasive test. ${ }^{36}$

The invasive strategy is indicated for patients with highrisk criteria, with coronary angiography performed within 72 hours (preferably up to 24 hours if there are multiple risk factors) and subsequent revascularization, if necessary. High-risk ischemic patients with refractory angina, associated with heart failure, arrhythmias, or hemodynamic instability should be considered for emergency coronary angiography ( $<2$ hours $){ }^{37}$

\section{Surgical Resource of Myocardial Revascularization}

Also known as bypass surgery, myocardial revascularization surgery is a procedure whereby the surgeon uses a segment of artery or vein to divert blood from the aorta to the coronary arteries. Most of these procedures are performed with cardiopulmonary bypass, that is, with the aid of equipment, which replaces the functions of the heart and lung during surgery. In some cases, it is performed with the patient's heart beating and the lung functioning normally. ${ }^{3}$ It presents a greater possibility of obtaining complete revascularization when compared to angioplasty. Its benefit deserves to be highlighted especially in those groups of patients with diabetes mellitus or with changes in ventricular function. ${ }^{3}$

\section{Conclusion}

Risk stratification and early diagnosis of these myocardial ischemic syndromes are essential conducts that must be taken without delay in the first 12 hours after hospital admission to avoid early mortality in the general population. Most deaths from AMI occur within the first hours of the event, and approximately $80 \%$ of cases occur within 24 hours of the event. Thus, it is essential that the entire propaedeutic, diagnostic and therapeutic structure necessary for individuals with these clinical conditions be available so that they are treated in an appropriate and timely manner.

Despite the incorporation of nuclear cardiology resources, modern and sophisticated equipment, new radiopharmaceuticals, and more advanced computer programs to support the care of coronary care units, a large part of these resources can only be found in large Brazilian cities, given the socio-economic and technological deficiency of the country's health system, which is unable to structure and organize the demand for this flow of patient care satisfactorily.

In our environment, the greatest difficulties faced by our hospitals and coronary urgency and emergency units is the unavailability of many of these indispensable diagnostic and therapeutic resources (equipment, devices, instruments, materials, specific medications, and qualified and trained labor), which often harms the correct risk stratification for the proper approach of patients with the suspected ACS, making the implementations of the protocols unfeasible.

This problem is a major challenge for Brazil, a country with continental dimensions that suffers from serious structural and operational bottlenecks. Despite the relevant cost-benefit aspect of the implemented protocols, the large-scale use of invasive and non-invasive methods described in this review article is restricted. It is observed that many of these resources presented are not yet available in many Brazilian regions, despite the great repressed demand for such procedures, which has aggravated the current situation and prevented comprehensive and 
Review Highlights

What Is Already Known?

ACS represents the main cause of death in the world, being among the pathologies with the greatest clinical and financial impact. Most cases of AMI are caused by the occlusion of a main coronary branch, being motivated by smoking, arterial hypertension, dyslipidemia, diabetes mellitus, in addition to a family history of early CAD (men $<55$ years old and women $<65$ years old).

\section{What Does This Study Add?}

This review article synthesizes the knowledge that ACS requires a critical medical approach with hospital diagnostic and therapeutic resources for assertive risk stratification and the respective selection of patients who are candidates for revascularization. The first step is the chest pain protocol with ECG, and for some patients, there are several methods proposed for stratification.

resolutive care for patients with acute coronary disease by the government, and because of a budgetary, technical, political, and geographical deficit. On the other hand, although in some places where such equipment and services are available, unfortunately, these cannot yet be considered routine procedures due to the inefficiency of the health system itself.

\section{Authors' Contributions}

DSTJ contributed to the drafting, literature review, article writing. Meanwhile, EMS and RCO contributed to the article writing and critical review. The authors read and approved the final version of the manuscript.

\section{Conflict of Interest Disclosures}

The authors declare that there is no conflict of interests.

\section{Ethical Approval}

Not applicable.

\section{References}

1. Silva FM, Pesaro AE, Franken M, Wajngarten M. Acute management of unstable angina and non-ST segment elevation myocardial infarction. Einstein (Sao Paulo). 2015;13(3):454461. doi:10.1590/s1679-45082015rw3172.

2. Lippi G, Favaloro EJ. Myocardial infarction, unstable angina, and white thrombi: time to move forward? Semin Thromb Hemost. 2019;45(1):115-116. doi:10.1055/s-0038-1657781.

3. Feitosa-Filho GS, Baracioli LM, Barbosa CJ, et al. SBC guidelines on unstable angina and non-ST-elevation myocardial infarction: executive summary. Arq Bras Cardiol. 2015;105(3):214-227. doi:10.5935/abc.20150118.

4. Eggers KM, Jernberg T, Lindahl B. Unstable angina in the era of cardiac troponin assays with improved sensitivity-a clinical dilemma. Am J Med. 2017;130(12):1423-1430.e1425. doi:10.1016/j.amjmed.2017.05.037.

5. Lei L, Bin Z. Risk factor differences in acute myocardial infarction between young and older people: a systematic review and meta-analysis. Int J Cardiovasc Sci. 2019;32(2):163 176. doi:10.5935/2359-4802.20190004.

6. Sandoval Y, Jaffe AS. Using high-sensitivity cardiac troponin T for acute cardiac care. Am J Med. 2017;130(12):1358-1365. e1. doi:10.1016/j.amjmed.2017.07.033.

7. Heusch G. Myocardial ischemia: lack of coronary blood flow myocardial oxygen supply-demand imbalance, or what? Am J Physiol Heart Circ Physiol. 2019;316(6):H1439-H1446. doi:10.1152/ajpheart.00139.2019.

8. Azab AE, Elsayed ASI. Acute myocardial infarction risk factors and correlation of its markers with serum lipids. J Appl Biotechnol Bioeng. 2017;3(4):385-391. doi:10.15406/ jabb.2017.03.00075.

9. Taban Sadeghi M, Mahmoudian B, Ghaffari S, et al. Value of early rest myocardial perfusion imaging with SPECT in patients with chest pain and non-diagnostic ECG in emergency department. Int J Cardiovasc Imaging. 2019;35(5):965-971. doi:10.1007/s10554-018-01518-0.

10. Truong QA, Schulman-Marcus J, Zakroysky P, et al. Coronary CT angiography versus standard emergency department evaluation for acute chest pain and diabetic patients: is there benefit with early coronary CT angiography? results of the randomized comparative effectiveness ROMICAT II Trial. J Am Heart Assoc. 2016;5(3):e003137. doi:10.1161/jaha.115.003137.

11. Franklin BA, Thompson PD, Al-Zaiti SS, et al. Exercise-related acutecardiovasculareventsand potential deleterious adaptations following long-term exercise training: placing the risks into perspective-an update: a scientific statement from the American Heart Association. Circulation. 2020;141(13):e705-e736. doi:10.1161/cir.0000000000000749.

12. Smith JN, Negrelli JM, Manek MB, Hawes EM, Viera AJ. Diagnosis and management of acute coronary syndrome: an evidence-based update. J Am Board Fam Med. 2015;28(2):283 293. doi:10.3122/jabfm.2015.02.140189.

13. Asrress KN, Williams R, Lockie $T$, et al. Physiology of angina and its alleviation with nitroglycerin: insights from invasive catheter laboratory measurements during exercise. Circulation. 2017;136(1):24-34. doi:10.1161/circulationaha.116.025856.

14. den Uil CA, Brugts JJ. Impact of intravenous nitroglycerin in the management of acute decompensated heart failure. Curr Heart Fail Rep. 2015;12(1):87-93. doi:10.1007/s11897-014-0230-8.

15. Bavry AA, Gong Y, Handberg EM, Cooper-DeHoff RM, Pepine CJ. Impact of aspirin according to type of stable coronary artery disease: insights from a large international cohort. Am J Med. 2015;128(2):137-143. doi:10.1016/j.amjmed.2014.09.028.

16. Sueta D, Tabata N, Hokimoto S. Clinical roles of calcium channel blockers in ischemic heart diseases. Hypertens Res. 2017;40(5):423-428. doi:10.1038/hr.2016.183.

17. Palmerini T, Benedetto $U$, Bacchi-Reggiani L, et al. Mortality in patients treated with extended duration dual antiplatelet therapy after drug-eluting stent implantation: a pairwise and Bayesian network meta-analysis of randomised trials. Lancet. 2015;385(9985):2371-2382. doi:10.1016/s01406736(15)60263-X

18. Lin TT, Lai HY, Chan KA, Yang YY, Lai CL, Lai MS. Single and dual antiplatelet therapy in elderly patients of medically managed myocardial infarction. BMC Geriatr. 2018;18(1):86 doi:10.1186/s12877-018-0777-4.

19. de Andrade PB, Borges LS. Antiplatelet agents in acute coronary syndromes. Int J Cardiovasc Sci. 2017;30(5):442-451. doi:10.5935/2359-4802.20170058.

20. Han Y, Guo J, Zheng Y, et al. Bivalirudin vs heparin with or without tirofiban during primary percutaneous coronary intervention in acute myocardial infarction: the BRIGHT 
randomized clinical trial. JAMA. 2015;313(13):1336-1346. doi:10.1001/jama.2015.2323.

21. He $\mathrm{P}$, Liu $Y$, Wei $X$, et al. Comparison of enoxaparin and unfractionated heparin in patients with non-ST-segment elevation acute coronary syndrome undergoing percutaneous coronary intervention: a systematic review and metaanalysis. J Thorac Dis. 2018;10(6):3308-3318. doi:10.21037/ jtd.2018.05.113.

22. de Matos Soeiro A, de Barros E Silva PGM, de Castro Roque EA, et al. Fondaparinux versus enoxaparin-which is the best anticoagulant for acute coronary syndrome?-Brazilian registry data. Arq Bras Cardiol. 2016;107(3):239-244. doi:10.5935/ abc. 20160127.

23. Casella IB, Puech-Leão P. Generic versus branded enoxaparin in prophylaxis and treatment of vein thrombosis. Rev Assoc Med Bras (1992). 2015;61(1):44-50. doi:10.1590/18069282.61.01.044.

24. Liu Z, Silvain J, Kerneis M, et al. Intravenous enoxaparin versus unfractionated heparin in elderly patients undergoing primary percutaneous coronary intervention: an analysis of the randomized ATOLL trial. Angiology. 2017;68(1):29-39. doi:10.1177/0003319716629541.

25. Montalescot G, Sabatine MS. Oral dual antiplatelet therapy: what have we learnt from recent trials? Eur Heart J. 2016;37(4):344-352. doi:10.1093/eurheartj/ehv377.

26. Roffi M, Patrono C, Collet JP, et al. 2015 ESC guidelines for the management of acute coronary syndromes in patients presenting without persistent ST-segment elevation: task force for the management of acute coronary syndromes in patients presenting without persistent ST-segment elevation of the European Society of Cardiology (ESC). Eur Heart J. 2016;37(3):267-315. doi:10.1093/eurheartj/ehv320.

27. Collet C, Capodanno D, Onuma Y, et al. Left main coronary artery disease: pathophysiology, diagnosis, and treatment. Nat Rev Cardiol. 2018;15(6):321-331. doi:10.1038/s41569-0180001-4.

28. Sabatine MS, Giugliano RP, Keech AC, et al. Evolocumab and clinical outcomes in patients with cardiovascular disease. $N$ Engl J Med. 2017;376(18):1713-1722. doi:10.1056/ NEJMoa1615664.

29. Eijsvogels TM, Maessen MF. Exercise for coronary heart disease patients: little is good, more is better, vigorous is best.
J Am Coll Cardiol. 2017;70(14):1701-1703. doi:10.1016/j. jacc.2017.08.016.

30. Dilsizian V, Bacharach SL, Beanlands RS, et al. ASNC imaging guidelines/SNMMI procedure standard for positron emission tomography (PET) nuclear cardiology procedures. J Nucl Cardiol. 2016;23(5):1187-1226. doi:10.1007/s12350-0160522-3.

31. Collet $\mathrm{C}$. Evidence based decision making between $\mathrm{PCl}$ and CABG. Arq Bras Cardiol. 2019;112(5):524-525. doi:10.5935/ abc. 20190076.

32. Tegn $\mathrm{N}$, Abdelnoor $\mathrm{M}$, Aaberge $\mathrm{L}$, et al. Invasive versus conservative strategy in patients aged 80 years or older with nonST-elevation myocardial infarction or unstable angina pectoris (After Eighty study): an open-label randomised controlled trial. Lancet. 2016;387(10023):1057-1065. doi:10.1016/s01406736(15)01166-6.

33. Bach RG, Cannon CP, Giugliano RP, et al. Effect of simvastatinezetimibe compared with simvastatin monotherapy after acute coronary syndrome among patients 75 years or older: a secondary analysis of a randomized clinical trial. JAMA Cardiol. 2019;4(9):846-854. doi:10.1001/jamacardio.2019.2306.

34. Arnett DK, Blumenthal RS, Albert MA, et al. 2019 ACC/AHA guideline on the primary prevention of cardiovascular disease: a report of the American College of Cardiology/American Heart Association Task Force on Clinical Practice Guidelines. Circulation. 2019;140(11):e596-e646. doi:10.1161/ cir.0000000000000678.

35. Naqvi SH, Abbas T, Tun HN, Naqvi AA, Zaffar Z, Gill BA, et al. Diagnostic accuracy of TIMI versus GRACE score for prediction of death in patients presenting with acute Non-ST Elevation Myocardial Infarction (NSTEMI). J Cardiol Cardiovasc Med. 2019;4:1-5. doi:10.29328/journal.jccm.1001032.

36. Littnerova S, Kala P, Jarkovsky J, et al. GRACE Score among six risk scoring systems (CADILLAC, PAMI, TIMI, Dynamic TIMI, Zwolle) demonstrated the best predictive value for prediction of long-term mortality in patients with ST-elevation myocardial infarction. PLoS One. 2015;10(4):e0123215. doi:10.1371/ journal.pone.0123215.

37. Stone GW, Sabik JF, Serruys PW, et al. Everolimus-eluting stents or bypass surgery for left main coronary artery disease. N Engl J Med. 2016;375(23):2223-2235. doi:10.1056/NEJMoa1610227 